\title{
Entamoeba histolytica
}

\author{
Adrienne J. Showler MD, Andrea K. Boggild MD MSc
}

\section{The gastrointestinal pathogen Ent- amoeba histolytica causes amoebiasis}

Clinical severity ranges from the asymptomatic passage of cysts in the stool to fulminant dysentery. Potentially fatal extraintestinal amoebiasis, including amoebic liver abscess, complicates $1 \%-$ $3 \%$ of infections. ${ }^{1}$ Pregnancy, immunocompromise, corticosteroid use, alcohol abuse and diabetes are risk factors for severe disease. ${ }^{2}$ Transmission can be sexual or occur via fecal contamination of food and water. ${ }^{3}$ Most infections are acquired abroad in tropical and subtropical areas, ${ }^{4}$ although household contacts of a patient with this infection, men who have sex with men and residents of institutions are also at risk. ${ }^{3}$

Infection with $E$. histolytica requires treatment; colonization with E. dispar does not

Entamoeba histolytica is a nationally and provincially notifiable disease; E. dispar is a harmless commensal with no public health implications. Colonization of the stool with E. dispar suggests potential exposure to other pathogens with fecal-oral transmission but does not cause diarrhea.

\begin{abstract}
Standard stool examinations for ova and parasites cannot reliably distinguish pathogenic E. histolytica from nonpathogenic Entamoeba dispar

Stool microscopy reports typically state "Entamoeba histolytica/dispar present." Although morphologically identical to E. histolytica, E. dispar is a nonpathogenic intestinal amoeba. False-negative result rates for stool microscopy approach $40 \%$, but decrease to $5 \%-15 \%$ with 3 or more specimens examined. ${ }^{1}$ Testing to confirm $E$. histolytica by polymerase chain reaction or enzyme immunoassay should be done on a separate, fresh and unpreserved specimen. ${ }^{5}$ In developed countries, the prevalence of E. histolytica/E. dispar is $2.4 \%$ among immigrants, $4 \%$ among travellers and $27 \%$ among men who have sex with men. ${ }^{4}$ Most cases represent $E$. dispar colonization; in 1 Canadian study, only $4.6 \%$ of cases involved E. histolytica. ${ }^{4}$
\end{abstract}

\section{Empiric treatment is rarely warranted}

Current guidelines recommend treatment only when E. histolytica is identified, or if dysentery of unknown cause persists despite empiric treatment of Shigella with consecutive 2-day courses of different antibiotic agents. ${ }^{6}$ Suspicion of amoebic liver abscess based on epidemiology and characteristic imaging should prompt empiric treatment. $^{2}$ Invasive disease requires dual
therapy

Symptomatic amoebiasis requires a 2-drug regimen: an amoebicidal agent, such as metronidazole, and a luminal-acting cysticidal agent, such as iodoquinol. ${ }^{7}$ A recent metaanalysis concluded that monotherapy is inadequate for most cases of amoebiasis. ${ }^{7}$ Asymptomatic cyst passage is treated with a luminal cysticide to prevent transmission and progression to invasive disease. A summary of therapeutic options is shown in Appendix 1 (available at www.cmaj.ca/lookup/suppl/doi :10.1503/cmaj.121576/-/DC1).

5. World Gastroenterology Organisation global guidelines. Acute diarrhea in adults and children: a global perspective. Milwaukee (WI): the Organisation; 2012. Available: www.worldgastroenterology .org/acute-diarrhea-in-adults.html (accessed 2013 Feb. 8).

6. Garcia LS, Smith JW, Fritsche TR. Cumitech 30A - selection and use of laboratory procedures for diagnosis of parasitic infections of the gastrointestinal tract. Garcia LS, coordinating editor. Washington (DC): ASM Press; 2003.

7. Gonzales MLM, Dans LF, Martinez EG. Antiamoebic drugs for treating amoebic colitis. Cochrane Database Syst Rev 2009;(2):CD006085.
Affiliations: Department of Medicine (Showler, Boggild), University of Toronto; Public Health Ontario Laboratories (Boggild), Public Health Ontario; Tropical Disease Unit, Division of Infectious Diseases (Boggild), University Health Network Toronto General Hospital, Toronto, Ont.

Correspondence to: Andrea K. Boggild, andrea .boggild@utoronto.ca

CMAJ 2013. DOI:10.1503/cmaj.121576 